\title{
Nonparametric, Low Bias, and Low Variance Time-Frequency Analysis of Myoelectric Signals
}

\author{
Kristin A. Farry, NASA/Johnson Space Center/ER, Houston, Texas 77058 USA \\ Richard G. Baraniuk and Ian D. Walker, Electrical and Computer Engineering, Rice University, \\ Houston, Texas 77251-1892 USA
}

Abstract. We apply Thomson's multiple window method to myoelectric signal time-frequency analysis for the first time. We extend Thomson's spectral estimation methodology for stationary signals to a time-frequency analysis tool, which we call the short-time Thomson transform. We compare this time-frequency analysis approach with the short-time Fourier transform.

Introduction. The myoelectric signal is, in general, non-stationary during all phases of force production. In a steady-state contraction, the non-stationarity is due to physiological variations such as blood flow and build-up of chemical byproducts of the muscle contraction process which affect action potential conduction velocity. During the initiation of a motion, the force and motion-dependent motor unit recruitment and firing rate modulation contributes non-stationarity [1].

Consequently, joint time-frequency methods, where we estimate the signal's spectrum locally at time intervals, appear well-suited to myoelectric signal analysis. Here, we compare two time-frequency methods suitable for piece-wise stationary signals in this work: one based on the well-known periodogram and one based on the newer Thomson's multiple window method (MWM) [2].

Methods. Hannaford and Lehman [3] first used short-time Fourier transforms (STFTs) to analyze the myoelectric signals recorded during rapid head and wrist movements. They used a Hamming-windowed STFT to compute the spectrogram

$$
\dot{P}_{h p}(f, m)=\left|\sum_{n=0}^{N-1} w[m-n] x[n] e^{-j 2 \pi f n}\right|^{2},
$$

where $x[n]$ is the myoelectric signal of length greater than the window length $N, w[n]$ is the Hamming window, $n$ is the time index inside the window, $m$ is the frequency-localization variable, and $f$ is frequency. They reported consistent patterns in the spectrograms of rapid movements in terms of mean frequency drift. This convinced us to try Hamming STFTs to compute motion classification features in a myoelectric control system [4]; however, the STFT's high variance resulted in unsatisfactory classification performance and led us to seek a lower variance time-frequency method.

Thomson's MWM provides a lower variance kernel. The short-time Thomson transform (STTT), introduced in [4] and [5], is:

$$
\hat{P}_{m w m}(f, m)=\frac{1}{2 N W} \sum_{k=0}^{2 N W W-1} \frac{1}{\lambda_{k}}\left|x_{k}(f, m)\right|^{2},
$$

where $x_{k}(f)$ are the eigenspectra

$$
\left|x_{k}(f, m)\right|^{2}=\left|\sum_{n=0}^{N-1} x[n] v_{k}[m-n] e^{-j 2 \pi f n}\right|^{2} .
$$

$v_{k}[n]$ is the $k$ th discrete prolate spheroidal sequence window and $\lambda_{x}$ is its corresponding eigenvalue [2]. $W$ is the resolution of the estimate. There are two versions of the STTT; these equations and results are for the cruder (and hence lower computation) version.

Results and Discussion. Figure 1 compares the Hamming-windowed STFT with the crude STTT $(N W=2)$ for a surface-measured Abductor Pollicis Longus and Extensor Pollicis Brevis monopolar myoelectric signal $6 \mathrm{~mm}$ recessed wet electrodes, bandpass filtered 3-300 Hz, sampled at 1000 $\mathrm{Hz}$ ). The STTT shows behavior closer to what our understanding of the muscle physiology leads us to expect: progressively more activity in the lower spectrum (below 75 $\mathrm{Hz}$ ) as more motor units are recruited to meet increasing force demands, coupled with upper spectrum activity from their action potentials, modulo tissue filtering [1].

Figure 2 shows a 3-dimensional view of the two approaches. Figure 3 superimposes 5 spectral time slices (10 ms apart) from the STFT and the STTT, covering a $50 \mathrm{~ms}$ interval just after the motion's start. We expect some change in the myoelectric spectra with increasing force demands; however, the STFT shows much greater changes over the same time interval than the STTT. The difference is the STFT's greater inherent variance.

Conclusions. The STTT results show much less time-smearing and variance than the STFT results. They suggest that the myoelectric spectrum may be more stationary than the STFT results indicate.

Acknowledgements. Support for this work included a NSF Graduate Fellowship, a Zonta International Fellowship, and two NSF Grants (MSS9024391 and MIP9457438). NASA/JSC provided funding (NAG9-740) and research equipment.

\section{References}

1. J. Basmajian and C. DeLuca "Muscles Alive," (Baltimore: Williams and Wilkins, 1985), 5th Ed.

2. D. Thomson. "Spectrum Estimation and Harmonic Analysis," Proc. of the IEEE, Vol. 70, No. 9, pp. 1055-1096. 
3. B. Hannaford and S. Lehman. "Short-Time Fourier Analysis of the Electromyogram: Fast Movements and Constant Contraction," IEEE Trans. on Biomedical Eng., BME-33, No. 12, pp. 1173-1181.

4. K. Farry. Issues in Myoelectric Teleoperation of Complex
Artificial Hands, Ph.D Dis., Elec. and Comp. Eng., Rice Univ., Nov 1994.

5. G. Frazer and B. Boashash. "Multiple Window Spectrogram and Time-Frequency Distributions," Signal Processing Research Centre, QUT, P.O. Box 2434, Brisbane 4001, Austrailia, 1994.
Figure 1: A sample thumb abduction myoelectric signal (top) and its STFT (middle) and STTT (bottom). The thumb motion becomes measureable at $0.15 \mathrm{sec}$.
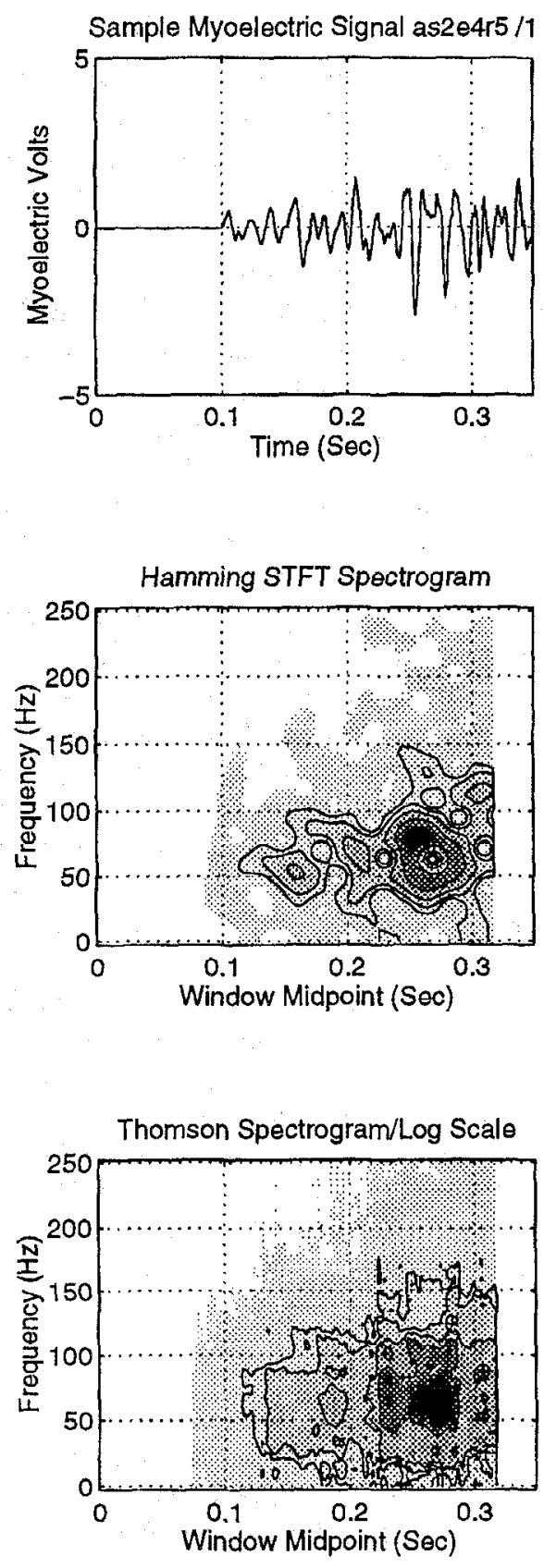

Figure 2: Three-dimensional views of the same signal's STFT (top) and STTT (bottom), showing the difference in variance and bias.

STFT

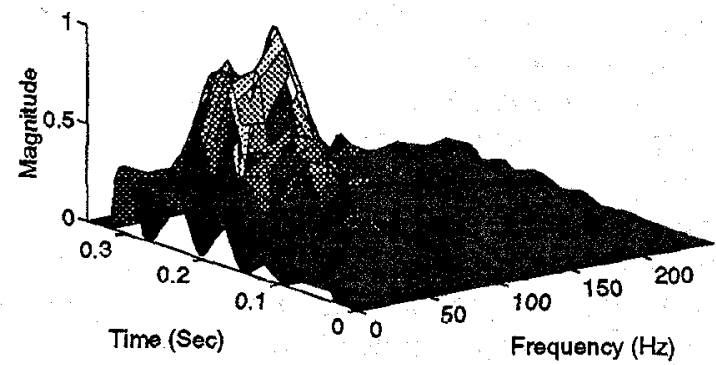

STT

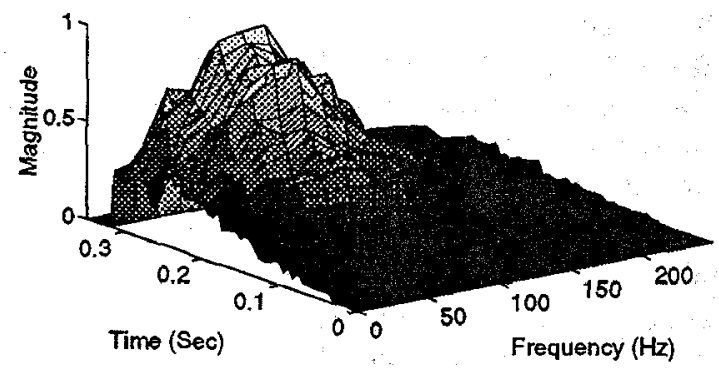

Figure 3: Five $64 \mathrm{~ms}$ time slices (spaced $10 \mathrm{~ms}$ apart) from the sample signal's STFT (left) and STTT (right), covering $50 \mathrm{~ms}$ just after motion start, superimposed.
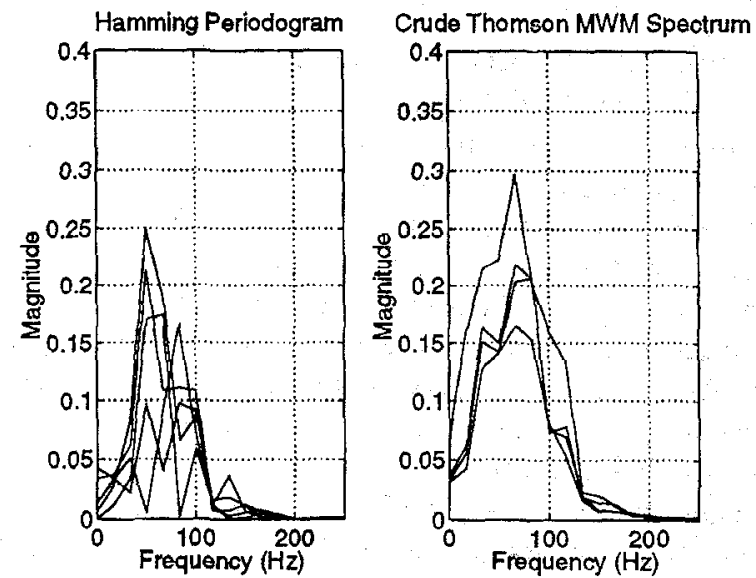